\title{
The importance of developmental mechanisms in understanding adolescent depression
}

\author{
Meg J. Dennison ${ }^{1}$
}

Received: 16 December 2015/Accepted: 23 March 2016/Published online: 13 April 2016

(C) Springer-Verlag Berlin Heidelberg 2016

The risk for developing depression markedly increases during the second decade of life [1]. Over the past two decades, considerable research has examined the unique characteristics of adolescence that confer risk for depression, spanning neurobiological, genetic, hormonal, behavioral and psychosocial domains. Identifying etiological mechanisms to guide effective prevention and intervention has proved challenging for many reasons, including the necessary reliance on observational rather than experimental studies, the high cost and attrition problems associated with longitudinal investigations and the integration of rapidly emerging fields, often accompanied with highly technical methods, such as genomics and functional neuroimaging. The heterogeneous classification of depressionrelated disorders, combined with high levels of comorbidity, has presented additional challenges to the study of contributing mechanisms, particularly if depression is a disorder with multiple underlying causes.

In "The ROOTS study ten years on: a review of findings on adolescent depression and future directions, challenges, and recommendations for longitudinal research" [2] the authors describe a multi-wave and multimodal population study of teenagers tracked from 14 to 21 years of age, involving assessment of psychosocial, biological and childhood adversity measures. The study has made a number of noteworthy contributions. The nuanced characterization of the relationship between adversity and

This comment refers to the article available at doi: 10.1007/s00127$015-1150-y$

Meg J. Dennison

megjd@uw.edu

1 Department of Psychology, University of Washington, UW Box 351525, Seattle, WA 98105, USA mental health helps identify sub-populations at greater risk and suggests unique risk pathways. Evidence that cognitive biases contribute to associations between genetic variation, stress exposure and mental health problems, as well as failure to show physical activity is an effective intervention for depression during adolescence, contributes to the search for modifiable targets for intervention. Description of developmental pathways to depression-for example, that elevated cortisol and moderate depressive symptoms in early adolescence predict later onset of clinical disorder, is useful for identifying underlying mechanisms and biomarkers for early detection. Finally, identification of an underlying transdiagnostic risk factor for mental disorders may assist in identifying common mechanisms that increase general susceptibility for psychiatric disorders and contribute to co-morbidity. Methodological strengths of the study that address the aforementioned challenges are the overall prospective design, use of nested studies to study distinctive subgroups, and data analysis techniques that integrate both person-centered and variable-centered perspectives, which allow observation of underlying mechanisms that may not initially appear intuitive.

Although epidemiological evidence [1] and neurodevelopmental changes [3] provide the impetus to focus on the period of adolescence as the crucible for mood disorders, it is unlikely that all contributing factors emerge only during this period of development. Instead, for many youth, it is likely that by adolescence the twig is bent, so is the tree inclined. For example, structural differences in the hippocampus are already observable among 12-year-old children who subsequently develop first-onset depression in adolescence [4]. Within the ROOTS study, elevated morning cortisol combined with sub-threshold depression symptoms already present at 14 years predicted clinical depression at 17 years. This discovery has immense 
clinical utility for early detection, and invites further investigation into whether this hormonal biomarker is present from birth or is an environmentally sensitive biomarker. Identifying when such vulnerabilities emerge provides clues as to the underlying developmental patterns that ultimately culminate in depression.

The importance of developmentally meaningful, longitudinal designs cannot be understated. Our knowledge of the optimal developmental windows in which to begin our studies has been a shifting target. For example, although extensive research has examined the role of puberty (focusing primarily on gonadarche) in heightening risk for emotional and behavioral problems, recent evidence suggests that timing of adrenarche-an earlier phase of sexual maturation-may be an important risk factor for current and future mental health problems [5]. Furthermore, given that the timing of depression onset spans a broad and dynamic period of human development, investigations should be informed by evidence that the etiology of depression probably varies with timing of onset [6]. Thus, the choice of when to study the disorder may lead to conflicting findings about the relative importance of particular etiological factors.

One important design issue in the ROOTS study is that children with depression onset prior to the start of study were not excluded; even though the prevalence of childhood depression is low, some children may have already experienced a depressive episode by the time they were enrolled in the study [1]. Although statistically controlling for baseline symptoms is one way of addressing this problem, without knowing the influence of prior illness on other putative variables of interest, the investigation of the factors contributing to onset becomes indistinguishable from possible effects arising from depression itself. The authors acknowledge this problem when describing difficulties disentangling the unique effects of adversity, psychopathology, and normative brain development on a cross-sectional measure of brain structure in later adolescence [2]. Truly developmental designs are needed for identifying developmental pathways that contribute to the onset of depression during adolescence.

At the population level, beginning in adolescence females experience a disproportionate degree of risk for depression [1], and reasons for this are not well characterized. A notable strength of the ROOTS study is the consistent exploration of sex-related patterns, including how sex moderates associations between risk factors and psychopathology. The finding that sex is a more important risk factor for adolescents exposed to low stress environments compared to high stress environments during childhood may suggest at least two unique, sex-differentiated pathways to psychopathology. This interesting finding may implicate sex-based developmental variables, such as pubertal timing, which are associated with both early life stress and risk for adolescent psychopathology in sexually differentiated ways [7]. The sex patterns described in the ROOTS study provides a forceful argument for continued consideration of sex-related differences in etiological pathways to adolescent depression.

The link between early life stress (ELS) and elevated risk for mental health problems in adolescence is well established; yet greater specificity regarding the nature of this association is needed to inform early intervention and treatment. Understanding specific effects is critical given that children exposed to ELS experience increased risk for both co-morbidity and resistance to conventional therapies [8]. The ROOTS study describes unique associations between adversity type and timing with risk for later psychopathology, challenging the view that the effects of different types of adversity are created equal. Sheridan and McLaughlin [9] have recently proposed that making distinctions between environments characterized by threat and violence versus those characterized by deprivation and neglect may help us more accurately differentiate the neurobiological pathways associated with different forms of adversity, which in turn will help us better understand developmental mechanisms linking adversity with psychopathology. Conceptual models of core underlying dimensions of environmental experience that influence development in unique ways are needed to guide the classification of adversity. Given the high co-occurrence of adverse experiences, other multivariate techniques are not likely to shed light on specificity.

An accurate taxonomy for describing the structure of psychopathology is critical to the identification of underlying mechanisms that can act as targets for effective intervention. The shift away from a categorical view of depression toward a dimensional view of psychopathology is supported by evidence from the ROOTS study. Their findings show psychotic phenomena and depression experienced during adolescence exist on a latent continuum of common mental distress, with psychosis representing greater severity. This evidence informs how we conceptualize disorders and, a practical level, highlights the increasing need for clinical research to be collaborative across disorder subtypes, particularly given underlying mechanisms and effective treatments identified for one disorder may be eligible for translation to others.

Advances in statistical methods over the past decade have been rapid, allowing researchers to test complex developmental and multi-factorial models, while also facilitating data-driven exploration using person-centered techniques such as latent class analysis. These sophisticated statistical approaches assist in identifying latent associations within large cohort data sets, driving exploration of novel connections between developmental, 
biological and psychosocial risk factors, with the ultimate aim of discovering of etiological mechanisms. Another recent approach involves examining how the covariance or "coupling" between two variables is moderated by a third variable. This technique is being used to identify and understand system-level effects, particularly biological systems such as brain connectivity and neuroendocrine function, which may be disrupted during development to create risk for psychopathology. For example, Ameis et al. [10] showed that while amygdala volume alone was not correlated with externalizing behaviors, an orbitofrontal cortex-amygdala network predicted rates of externalizing behavior in children and adolescents.

In sum, the ROOTS study has made an important contribution to the study of adolescent depression, describing a number of novel links between depression and risk factors operating at multiple levels of influence, shedding light on who is at risk, as well as identifying and eliminating plausible targets for intervention. This work confirms that the study of interactions between persons and their environments across developmental periods, rather than discrete characteristics such as isolated genes, are necessary for building mechanistic models of psychopathology. Continued focus on tracking patterns of development prior to the onset of depression will allow discernment of unique pathways that result in depression during adolescence. Identification of these developmental mechanisms creates an opportunity for early and targeted intervention, with the potential to disrupt the developmental course of this highly recurrent, chronic and disabling disease.

\section{Compliance with ethical standards}

Conflict of interest The author declares that she has no conflicts of interest.

\section{References}

1. Kessler RC, Avenevoli S, Ries Merikangas K (2001) Mood disorders in children and adolescents: an epidemiologic perspective. Biol Psychiatry 49(12):1002-1014
2. Lewis G, Jones PB, Goodyer I (2016) The ROOTS study: a 10-year review of findings on adolescent depression, and recommendations for future longitudinal research. Soc Psychiatry Psychiatr Epidemiol 51(2):161-170. doi:10.1007/s00127-0151150-y

3. Paus T, Keshavan M, Giedd JN (2008) Why do many psychiatric disorders emerge during adolescence? Nat Rev Neurosci 9(12):947-957

4. Whittle S, Lichter R, Dennison M, Vijayakumar N, Schwartz O, Byrne ML, Simmons JG, Yucel M, Pantelis C, McGorry P, Allen NB (2014) Structural brain development and depression onset during adolescence: a prospective longitudinal study. Am J Psychiatry 171(5):564-571. doi:10.1176/appi.ajp.2013.13070920

5. Mundy LK, Romaniuk H, Canterford L, Hearps S, Viner RM, Bayer JK, Simmons JG, Carlin JB, Allen NB, Patton GC (2015) Adrenarche and the emotional and behavioral problems of late childhood. J Adolesc Health 57(6):608-616. doi:10.1016/j.jado health.2015.09.001

6. Jaffee SR, Moffitt TE, Caspi A, Fombonne E, Poulton R, Martin J (2002) Differences in early childhood risk factors for juvenileonset and adult-onset depression. Arch Gen Psychiatry 59(3):215-222

7. Negriff S, Saxbe DE, Trickett PK (2015) Childhood maltreatment, pubertal development, HPA axis functioning, and psychosocial outcomes: an integrative biopsychosocial model. Dev Psychobiol 57(8):984-993. doi:10.1002/dev.21340

8. Shamseddeen W, Asarnow JR, Clarke G, Vitiello B, Wagner KD, Birmaher B, Keller MB, Emslie G, Iyengar S, Ryan ND, McCracken JT, Porta G, Mayes T, Brent DA (2011) Impact of physical and sexual abuse on treatment response in the Treatment of Resistant Depression in Adolescent Study (TORDIA). J Am Acad Child Adolesc Psychiatry 50(3):293-301. doi:10.1016/j. jaac.2010.11.019

9. Sheridan MA, McLaughlin KA (2014) Dimensions of early experience and neural development: deprivation and threat. Trends Cogn Sci 18(11):580-585. doi:10.1016/j.tics.2014.09.001

10. Ameis SH, Ducharme S, Albaugh MD, Hudziak JJ, Botteron KN, Lepage C, Zhao L, Khundrakpam B, Collins DL, Lerch JP, Wheeler A, Schachar R, Evans AC, Karama S (2014) Cortical thickness, cortico-amygdalar networks, and externalizing behaviors in healthy children. Biol Psychiatry 75(1):65-72. doi:10. 1016/j.biopsych.2013.06.008 